\title{
Linfedema como presentación inusual de cáncer de próstata
}

\author{
Ruiz Ruiz S*, Auguet Quintillà T*, Raventós Estellé A**, Florit Serra L*, Trobat Vanrell FX***, \\ Richart Jurado C*. \\ *Departamento de Medicina Interna. ${ }^{* *}$ Departamento de Anatomía Patológica. ${ }^{* * *}$ Servicio de Urología. \\ Hospital Universitari Joan XXIII de Tarragona. Universitat Rovira i Virgili.
}

Actas Urol Esp. 2007;31(3):289-291

\section{RESUMEN}

LINFEDEMA COMO PRESENTACIÓN INUSUAL DE CÁNCER DE PRÓSTATA

La etiología más frecuente del linfedema en nuestro medio es la tumoral y generalmente debido al tratamiento del mismo. Sin embargo la presentación inicial del cáncer de próstata como linfedema es muy poco frecuente, habiendo muy pocos casos descritos en la literatura. Exponemos el caso de un paciente que ingresó para estudio de linfedema en región genital y en extremidades inferiores siendo diagnosticado de cáncer de próstata con adenopatías retroperitoneales. El tratamiento de elección es el bloqueo hormonal, siendo el pronóstico muy pobre y la supervivencia escasa a los cinco años del diagnóstico.

Palabras clave: Linfedema. Carcinoma. Próstata.

\section{ABSTRACT}

LYMPHEDEMA AS AN INUSUAL PRESENTATION OF PROSTATE CARCINOMA.

The most common etiology of lymphedema in our environment is tumoral and, generally, it is due to treatment of the cancer itself. However, the initial presentation of prostate carcinoma as lymphedema is very rare. In fact, there aren't very few documented cases in literature. We described the case of a patient who was admitted into the hospital to be examined for lymphedema in his genitals and legs. Our patient was diagnosed of prostate carcinoma with retroperitoneal adenopathies. The best treatment in this case is block-hormonal therapy. Prognosis is very bad and the survival rate is poor within five years of diagnosis.

Keywords: Lymphedema. Carcinoma. Prostate.

$\mathrm{L}^{\mathrm{a}}$ a etiología más frecuente del linfedema en todo el mundo es la filariasis, pero en nuestro medio la causa más frecuente es la patología tumoral, bien debido a obstrucción del sistema linfático, o más comúnmente secundaria a los tratamientos realizados (cirugía y radioterapia). Las neoplasias de vejiga, próstata, recto y los linfomas son las más comúnmente implicadas ${ }^{1}$.

El linfedema como manifestación inicial de neoplasia prostática es muy infrecuente.

Presentamos un caso clínico que debutó con linfedema en ambas extremidades inferiores y genitales secundario a invasión ganglionar inguinal y retroperitoneal por cáncer de próstata.

\section{CASO CLÍNICO}

Varón de 62 años que consultó por edemas progresivos en ambas extremidades inferiores, región genital e inguinal de aproximadamente siete meses de evolución, sin ninguna otra clínica acompañante. Entre sus antecedentes sólo constaban hipertensión arterial y cólicos nefríticos no complicados.

En la exploración física llamaba la atención la presencia de edema de consistencia dura en zona púbica, incluyendo pene, escroto y región inguinal, que se extendía por ambas extremidades inferiores siendo más evidente en la izquierda, sin fovea. No existían lesiones cutáneas, ni ade- 
nopatías laterocervicales o axilares. Palpación de probables adenopatías inguinales.

Se realizó un tacto rectal que mostró una próstata aumentada de tamaño, dura y no dolorosa.

La analítica incluyendo bioquímica, hemograma, proteinograma, coagulación y estudio de orina con proteinuria de 24 horas que fue estrictamente normal. Se solicitaron marcadores tumorales destacando un PSA de 100 microgra$\operatorname{mos} / 1$.

Tanto la ecografía como el TC abdominal objetivaron múltiples adenopatías retroperitoneales paraaórticas y en ambas cadenas ilíacas así como una próstata aumentada de tamaño (Fig. 1). Se consultó con el Servicio de Urología y se llevó a cabo la biopsia prostática concluyente para adenocarcinoma de próstata (Fig. 2) y de la adenopatía inguinal que mostró invasión por adenocarcinoma prostático (Fig. 3). El estudio inmunohistoquímico puso de manifiesto una intensa positividad de la población neoplásica para la fosfatasa alcalina prostática, el antígeno prostático específico (PSA) y para los receptores de andrógenos (Fig. 4). La gammagrafía ósea mostró aumento de captación retroauricular y en apófisis espinosa y carilla auricular de C3.

Se inició tratamiento hormonal con antiandrógenos y análogos de LHRH. Ante la presencia de metástasis óseas, se añadió ácido zoledrónico. A los nueve meses del tratamiento, el paciente se

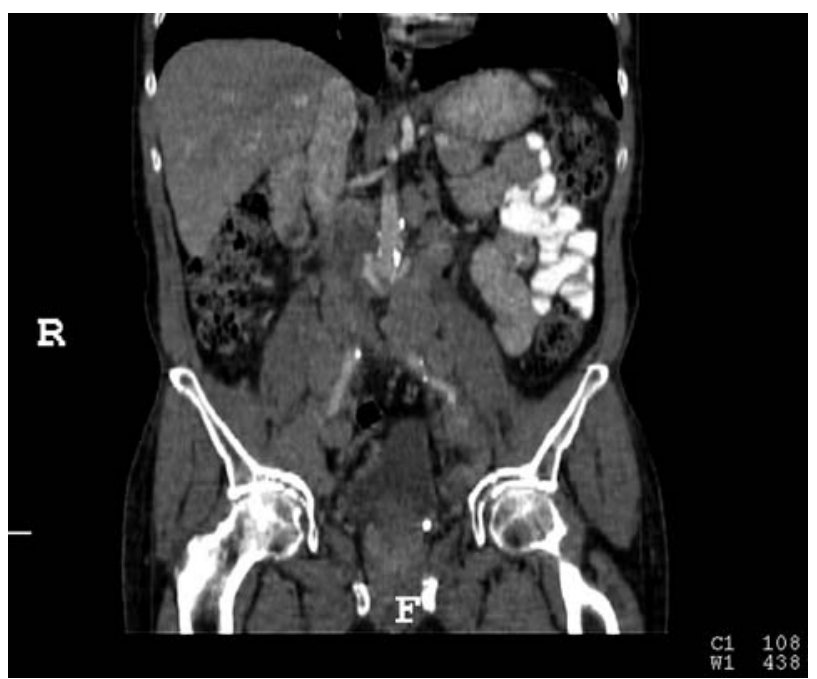

FIGURA 1. TAC abdominal. Corte sagital. Se objetivan adenopatias paraaórticas y en cadenas iliacas.

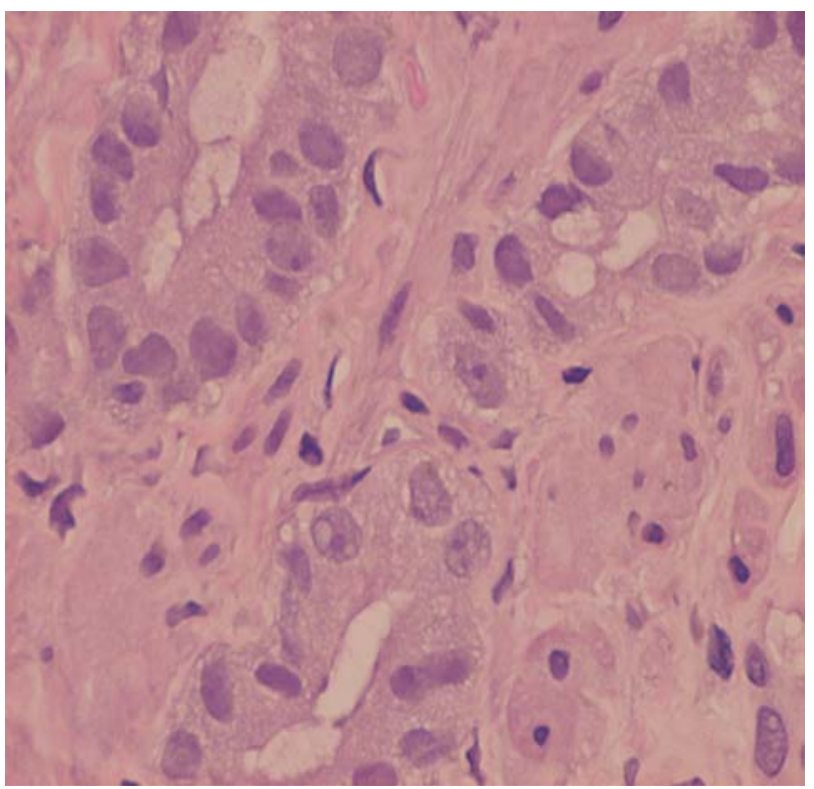

FIGURA 2. Biopsia de próstata. Estructuras glandulares constituidas por células cuboideas atípicas con nucleolo prominente (H-E $x$ 400)

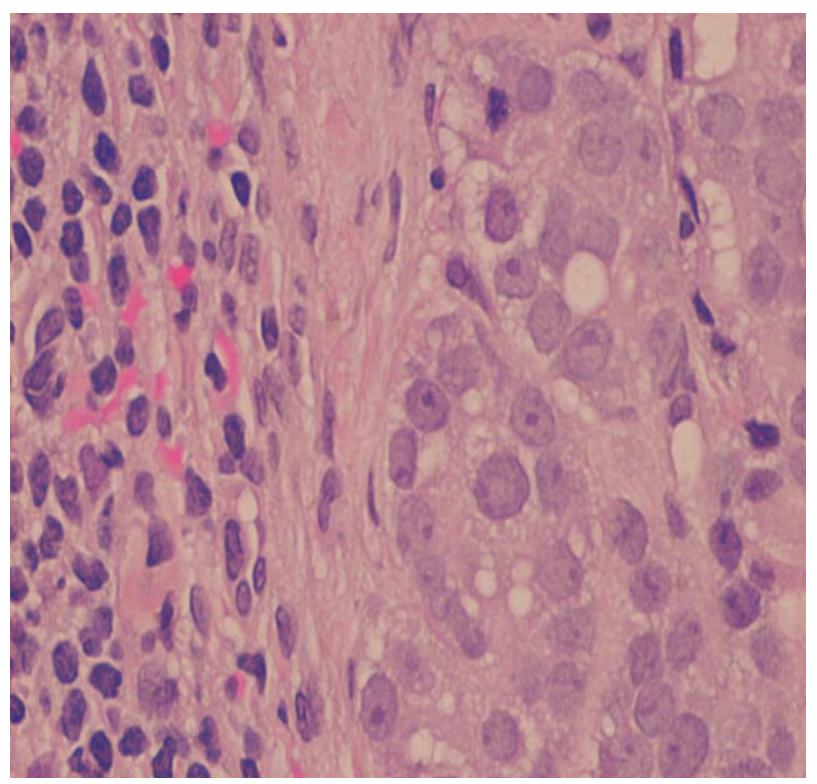

FIGURA 3. Ganglio linfático preservado (izquierda de la imagen) y metástasis por adenocarcinoma (H-E $x$ 400).

hallaba estable desde el punto de vista clínico y sin cambios en la exploración física. El PSA fue de 4,6 microgramos/1.

\section{DISCUSIÓN}

Los ganglios linfáticos son el asiento más frecuente de metástasis por adenocarcinoma prostático, seguido de los huesos y los pulmones. Sin 


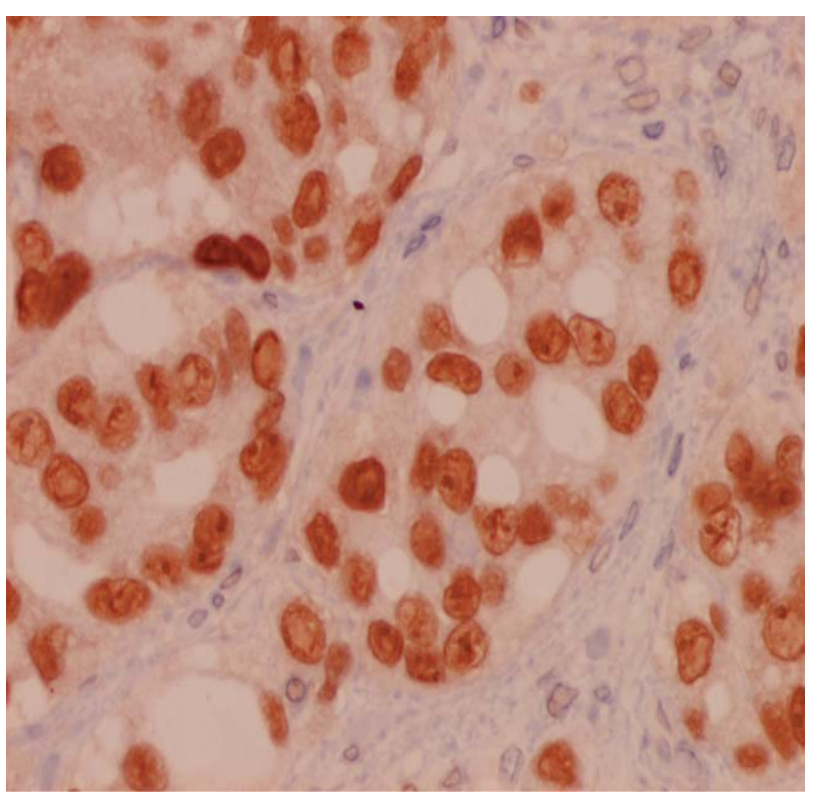

FIGURA 4. Técnica de inmunohistoquímica. Expresión nuclear para receptores de andrógenos en el adenocarcinoma.

embargo, el linfedema secundario a invasión ganglionar por adenocarcinoma de próstata es muy infrecuente ${ }^{2,3,4,7}$, sobretodo como forma de presentación inicial. Ello es debido a que el drenaje linfático de la próstata se realiza como primer escalón a nivel del obturador y de la cadena ilíaca. Es también importante señalar que la invasión tumoral de ganglios inguinales es infrecuente en el momento del diagnóstico ${ }^{3,6} \mathrm{del}$ adenocarcinoma de próstata, ya que suele aparecer años después del mismo.

Ante una neoplasia prostática con afectación ganglionar, como en el cáncer de próstata metastático, el bloqueo hormonal sigue siendo la base terapéutica estándar ${ }^{3,5,8}$. Sin embargo, algunos autores sugieren un tratamiento combinado con radioterapia coadyuvante ${ }^{3,5} \mathrm{o}$ añadir quimioterapia en pacientes muy concretos con cáncer de próstata de alto grado, ya que la supervivencia es escasa y la toxicidad sería significativa ${ }^{8,9}$. A pesar de ello, la supervivencia es inferior a cinco años en los casos descritos ${ }^{5}$. Nuestro paciente, en tra- tamiento con antiandrógenos y análogos de LHRH, así como ácido zoledrónico, se halla estable de su enfermedad a los nueve meses del diagnóstico.

Creemos que es interesante la comunicación de este caso, ya que, a pesar de su infrecuencia, el carcinoma de próstata debería incluirse en el diagnóstico diferencial del linfedema de extremidades inferiores y de región genital.

\section{REFERENCIAS}

1. Vignes S, Trévidic P. Lymphoedèmes pénoscrotaux: étude rétrospective de 33 cas. Ann Dermatol Venereol. 2005;132 (1):21-25.

2. Antony J, Reddy PS. An unusual presentation of carcinoma of the prostate. J Urology. 1985; 135(3):595-596.

3. Huang E, Teh BS, Mody DR, Carpenter LS, Butler EB. Prostate adenocarcinoma presenting with inguinal lymphadenopathy. Urology. 2003;61(2):463.

4. Ústündag Y, Yesilli C, Aydemir S, Savranlar A, Yazicioglu K. A life-threatening hematochesia after transrectal ultrasound-guided prostate needle biopsy in a prostate cancer case presenting with lymphedema. Int Urol Nephrol. 2004; 36(3):397-400.

5. Buskirk SJ, Pisansky TM, Atkinson EJ, Schild SE, O’Brien PC, Wolfe JT, et al. Lymph Node-Positive Prostate Cancer: Evaluation of the Results of the Combination of Androgen Deprivation Therapy and Radiation Therapy. Mayo Clin Proc. 2001;76(7):702-706.

6. Oyan B, Engin H, Yalcin S. Generalized lymphadenopathy: a rare presentation of disseminated prostate cancer. Med Oncol. 2002;19(3):177-179.

7. Llarena Ibarguren R, Zabala Egurrola JA, Arruza Echevarria A, Arregui Erbina P, Pertusa Pena C. Lymphatic involvement predominanting and revealing prostata carcinoma. Arch Esp Urol. 1992;45(5):469-471.

8. Grupo de expertos en cáncer de próstata. Cáncer de próstata de alto riesgo y diseminado (Documento de Consenso de Recomendaciones). Actas urológicas españolas. 2005;29(9): 826-827.

9. Bagley CM Jr, Lane RF, Blasko JC, Grimm PD, Ragde H, Cobb OE, et al. Adjuvant chemohormonal therapy of high risk prostate carcinoma. Ten years result. Cancer. 2002; 94(10):2728-2732.

Correspondencia autor: Dra. T. Auguet Quintanilla

Servicio Medicina Interna. Hospital U Joan XXIII. Tarragona.

Mallafré Guasch, 4. 43007 Tarragona

Tel.: 977-295833

E-mail autor: tauguet@hj23.icsagencat.net

Información articulo: Nota clínica

Trabajo recibido: enero 2006

Trabajo aceptado: marzo 2006 